Western University

Scholarship@Western

$10-11-2019$

\title{
Novel Green Micro-Synthesis of Graphene-Titanium Dioxide Nano- Composites with Photo-Electrochemical Properties
}

Nourwanda M. Serour

Alexandria University

Ahmed S.E. Hammad

Egypt-Japan University of Science and Technology

Ahmed H. El-Shazly

Alexandria University

Dina A. El-Gayar

Alexandria University

Shaaban A. Nosier

Alexandria University

Follow this and additional works at: https://ir.lib.uwo.ca/chemengpub

Part of the Biomedical Engineering and Bioengineering Commons, and the Chemical Engineering Commons

Citation of this paper:

Nourwanda M. Serour, Ahmed S.E. Hammad, Ahmed H. El-Shazly, Dina A. El-Gayar and Shaaban A. Nosier, "Novel Green Micro-Synthesis of Graphene-Titanium Dioxide Nano- Composites with Photo-

Electrochemical Properties", Current Nanoscience (2019) 15: 606. https://doi.org/10.2174/

1573413715666181212123137 


\section{Novel Green Micro-Synthesis of Graphene-Titanium Dioxide Nano-Composites with Photo- \\ Electrochemical Properties}

1) Nourwanda Mohamed Serour ${ }^{1 *}$, Ahmed Salah Eldin Hammad ${ }^{2,3}$, Ahmed Hassan El-Shazly ${ }^{1,2}$, Dina Ahmed El-Gayar', Shaaban Attia Nosier ${ }^{1}$

1 Department of Chemical Engineering, Faculty of Engineering, Alexandria University, Alexandria, Egypt

2 Department of Chemical and Petrochemicals Engineering, Egypt-Japan University of Science and Technology, EJUST, Alexandria, Egypt

3 Department of Chemical Engineering, Faculty of Engineering, Port Said University, Port Said, Egypt

Corresponding Author: Nourwanda Serour.

E-mail: eng-nourwanda.sourour@alexu.edu.eg

Phone: +20-122 1276464 .

Article Category: Research Article

Short Running Title: Novel Preparation of GTNC with Photo-electrochemical properties

Keywords: Micro-synthesis, graphene-titanium dioxide nanocomposite, chemical exfoliation, sol-gel, energy-dispersive Xray spectroscopy, chronoamperometry, linear sweep voltammetry. 


\begin{abstract}
Background: Graphene-Titanium dioxide nano-composite forms a very promising material in the field of photoelectrochemical research. Methods: In this study, a novel environment-friendly synthesis method was developed to produce well-distributed anatase nano-titanium dioxide spherical particles on the surfaces of graphene sheets, this novel method has great advantages over previously developed methods of producing graphene-titanium dioxide nanocomposites (GTNCs). High calcination temperature $650^{\circ} \mathrm{C}$ was used in the preparation nano titanium dioxide, chemical exfoliation for graphene synthesis and GTNC was produced by our novel method by depositing titanium dioxide nanoparticles on graphene sheets using a Y-shaped micro-reactor under a controlled pumping rate with minimal use of chemicals. Results: The physiochemical and crystallographic properties of the GTNC were confirmed by TEM, XRD, FTIR and EDX measurements, confirmed process repeatability. Spherical nano-titanium dioxide was produced in the anatase phase with very high crystallinity and small particle diameters ranging from $9 \mathrm{~nm}$ to $25 \mathrm{~nm}$, also the as prepared graphene (RGO) exhibited minimal flake folding and a high carbon content of $81.28 \%$ with a low oxygen-to-carbon atomic ratio of 0.172 and GTNCs produced by our novel method had a superior loading content, a homogeneous distribution and a $96.6 \%$ higher content of titanium dioxide particles on the graphene sheets compared with GTNCs prepared with the one-pot method. For its photoelectrochemical properties, chronoamperometry showed that GTNC sample (2) had a higher peak current of $60 \mu \mathrm{A}$ compared with that of GTNC sample (1), which indicates that the separation and transfer of electron-hole pairs are better in the case of GTNC sample (2) and according to the LSV results, the generation of photocurrent in the samples can be observed through multiple on-off cycles, which indicates that the electrodes are stable and that the photocurrent is quite reversible.
\end{abstract}




\section{Introduction}

Graphene loaded with titanium dioxide nanoparticles forms a very promising nanocomposite with superior physical, chemical and thermal properties. Such nanocomposites have made remarkable contributions in many industrial applications, such as those requiring the enhancement of photocatalytic activity, electrical conductivity, and heat transfer.

Graphene, which consists of a two-dimensional hexagonal crystal lattice of carbon atoms, was discovered in 2004 by Geim and Nocoselov [1]. Graphene proved to have a large specific surface area of approximately $2630 \mathrm{~m}^{2} / \mathrm{gram}$, an excellent thermal conductivity of $5000 \mathrm{~W} / \mathrm{m} . \mathrm{K}$, a band gap of zero and high photocatalytic activity due to its ability to absorb up to $2.3 \%$ of white light and due to the relaxation and recombination of photo-induced electron-hole pairs in graphene, which depends mainly on the carrier concentration [2]. The primary methods used to produce graphene are micromechanical exfoliation [3], liquid-phase exfoliation [4], chemical exfoliation [5-8] and chemical vapour deposition (CVD) [9, 10]. On the other hand, nano-titanium dioxide is a non-metallic compound that is found in three main crystal forms: rutile, anatase and brookite. Titanium dioxide has many distinguishing properties, such as its chemical stability, non-toxicity, highly efficient photocatalytic effect, high refractive index and heat resistance, and it is used to enhance heat transfer in many applications, such as pigments, photocatalysis and nanofluidic. There are several synthesis methods for nano-titanium dioxide, such as the sol-gel technique [11, 12], the sol technique [13], the hydrothermal technique [14], the solvothermal technique [15], thermal coprecipitation [16-19], sono-chemical synthesis [20], CVD [21] and physical vapour deposition. Other methods include the direct oxidation of titanium metal into titanium dioxide using either an oxidizing agent or anodization, electrodeposition or microwave methods [22]. Recently, graphene sheets tailored with nano-titanium dioxide nanoparticles have become an attractive material, especially for use in photocatalysis applications, due to the distinctive properties of both components. Such composites are favourable for photocatalytic applications due to the ease of photogeneration through the graphene material, which enhances the photocatalytic properties of the composites over those of titania nanoparticles alone due to the excellent electron trapping and transportation properties of graphene, resulting in the suppression of the photogenerated electron-hole recombination in titania [23]. The most common methods used to produce graphene-titanium dioxide nanocomposites (GTNCs) are the hydrothermal and solvothermal methods [24, 25]. However, they are very complicated, time consuming, and costly and require high chemical consumption. In 2015, Arun Kanti et al. [26] developed a cheap and eco-friendly method of producing GTNCs. Unfortunately, the produced composite had a very low loading content of nano-titanium dioxide on the graphene sheets, which is not desirable in photocatalysis applications [27]. Therefore, we developed a new modification to this process to enable the production of GTNCs with a homogeneous distribution and a higher loading content of nano-titanium dioxide on the graphene sheets. 
In this study, GTNCs were produced using two methods: the original green one-pot synthesis process, which results in a low content of titanium dioxide in the composite and a non-uniform distribution, and our new approach, in which the composite is produced using a micro-reactor. The new approach yields composites shows a higher loading and a uniform, homogeneous distribution of titania particles on the surfaces of the graphene sheets. Photo-electrochemical measurements, including linear scan voltammetry (LSV) and chronoamperometry (CAM), were performed on GTNC samples that were produced using both our novel modified system and the original method [26], and the results were evaluated to test the effects of our novel green micro-synthesis method on the photo-electrochemical properties of the produced composites.

\section{Materials and Methods}

\subsection{Materials and Equipment}

All reagents used were purchased from Sigma-Aldrich and of very high grade: titanium tetrachloride of $99.5 \%$ purity, sulfuric acid (98\%), ammonium hydroxide solution (33\% concentration), absolute ethanol (99.9\% concentration), graphite powder, nitric acid, hydrazine hydrate, methanol, hydrogen peroxide and potassium permanganate. Deionized water was freshly produced and directly used.

The equipment used was as follows: a heater and magnetic stirrer, a fuming hose, an ice bath, a pH meter, a vacuum dryer, a muffle furnace, a centrifuge, an ultrasonic probe (power, $500 \mathrm{~W}$; amplitude, 40\%; frequency, $20 \mathrm{kHz}$, Hielscher, Germany), an ultrasonic bath (power, $160 \mathrm{~W}$; frequency, $40 \mathrm{kHz}$ ), a drying oven, an agate mortar and pestle, a microwave irradiation reactor (power, $1000 \mathrm{~W}$ ), a syringe pump, a Y-shaped micro-reactor, field-emission transmission electron microscope (JEOL JEM-2100F,USA), X-ray diffractor (XRD-6100 Shimadzu, Japan) and Vertex 70 IR spectrometer (Bruker Scientific Instruments, Germany). All glassware used was rinsed thoroughly with soap and tap water and was then washed with deionized water immediately before use.

\subsection{Nano Particles Synthesis}

\subsubsection{Anatase Nano-Titanium Dioxide Synthesis via the Sol-Gel Method}

Spherical anatase nano-titanium dioxide was prepared via the sol-gel method with the hydrolysis of titanium tetrachloride as a precursor in the presence of sulfuric acid, as reported by Li et al. [28]. First, $150 \mathrm{ml}$ of deionized water was mixed with a solution of $10 \%$ diluted sulfuric acid. The mixture was placed in a beaker surrounded by an ice bath; then, magnetic stirring at speeds ranging from 1200 to $1500 \mathrm{rpm}$ was applied until the temperature of the solution reached $0^{\circ} \mathrm{C}$. Next, titanium tetrachloride was added in a dropwise manner under continuous stirring while the temperature was maintained at $0^{\circ} \mathrm{C}$, using a 1:0.4 molar ratio of $\mathrm{TiCl}_{4}$ to $10 \% \mathrm{H}_{2} \mathrm{SO}_{4}$ and a 1:15 volume ratio of $\mathrm{TiCl}_{4}$ to $\mathrm{H}_{2} \mathrm{O}$. After half an hour, the solution turned greyish white in colour as titanium oxy-sulphate formed, as described by the hydrolysis reaction in equation (1), and white 
fumes of $\mathrm{HCl}$ emerged. The solution was then heated at a hydrolysis temperature of $45^{\circ} \mathrm{C}$ until the solution became transparent again. Then, ammonium hydroxide solution was added in a dropwise manner until the $\mathrm{pH}$ became slightly higher than 7 , and a white precipitate started to form as nanoparticle formation was stimulated by the presence of an alkaline medium. The mixture was left to gel overnight. Then, the mixture was filtered, and the precipitate was washed with ethanol and distilled water using centrifugation at $6000 \mathrm{rpm}$ to remove any impurities, followed by drying in a vacuum oven to prevent contamination of the sample. The final step was calcination of the powder in a muffle furnace at $650^{\circ} \mathrm{C}$ for $90 \mathrm{~min}$, after which the sample was ground. The hydrolysis and calcination temperatures were increased to promote the formation of spherical anatase nano-titanium dioxide. The chemical reaction sequence occurred as follows:

Hydrolysis of $\mathrm{TiCl}_{4}$ to produce titanium oxy-sulphate:

$\mathrm{TiCl}_{4}+\mathrm{H}_{2} \mathrm{O}+\mathrm{H}_{2} \mathrm{SO}_{4} \rightarrow$ Ti-O-SO 4 (Grey sol.) $+4 \mathrm{HCl} \uparrow$ (white fumes)

Addition of ammonium hydroxide to neutralize the mixture, leading to the formation of a white precipitate:

$\mathrm{Ti}-\mathrm{O}-\mathrm{SO}_{4}+2 \mathrm{NH}_{4} \mathrm{OH} \rightarrow \mathrm{H}_{2} \mathrm{TiO}_{3} \downarrow+\left(\mathrm{NH}_{4}\right)_{2} \mathrm{SO}_{4}$

Formation of titanium dioxide after washing, drying and heating:

$\mathrm{H}_{2} \mathrm{TiO}_{3} \longrightarrow \mathrm{TiO}_{2} \downarrow$ (white ppt.) $+\mathrm{H}_{2} \mathrm{O}$

\subsubsection{Graphene (Reduced Graphene Oxide) Synthesis via Chemical Exfoliation}

The chemical exfoliation process consisted of two main steps. In the first step, the modified Hummers' method was used to produce graphene oxide (GO) from graphite flakes, and the second step was the reduction of the GO using hydrazine hydrate to obtain graphene (reduced graphene oxide, RGO). This modified synthesis process involves both the oxidation and exfoliation of graphite sheets due to the thermal treatment of the solution. The synthesis method is described in detail as follows. First, graphite flakes were added to $500 \mathrm{ml}$ of $\mathrm{H}_{2} \mathrm{SO}_{4}(98 \%)$ and $160 \mathrm{ml}$ of $\mathrm{HNO}_{3}$ in a $1000 \mathrm{ml}$ volumetric flask kept in an ice bath under continuous stirring. The mixture was then stirred for 1 hour at $0^{\circ} \mathrm{C}$, and potassium permanganate was added to the suspension very slowly at a rate of $1 \mathrm{gm} / \mathrm{min}$. The rate of addition was carefully controlled to keep the reaction temperature lower than $15^{\circ} \mathrm{C}$. The mixture was maintained under stirring for four days and subjected to ultrasonication for 20 min every day while adding ice cubes; the solution was finally treated with $40 \mathrm{ml}$ of $\mathrm{H}_{2} \mathrm{O}_{2}$, causing the colour to change to bright yellow. It was then left to sit without stirring for 3-4 hours to allow the particles to settle to the bottom, after which the remaining liquid was poured off, leaving the precipitate at the bottom. The resulting mixture was repeatedly washed using a centrifuge, first with $10 \% \mathrm{HCl}$ and then with deionized (DI) water, several times until it formed a gel-like substance (of neutral $\mathrm{pH}$ ). After centrifugation, the gel-like substance was vacuum dried at $60^{\circ} \mathrm{C}$ for more than 6 hours to obtain GO powder. In the reduction step, $2 \mathrm{ml}$ of hydrazine hydrate was added to the suspension (GO powder in distilled water) and 
heated at $80^{\circ} \mathrm{C}$ using microwaves. A kind of black flocculent substance gradually precipitated out of the solution. The product was filtered using qualitative filter paper. Finally, the resulting black product was washed with methanol and water and dried at $80^{\circ} \mathrm{C}$ for one day to obtain sheets of graphene, also known as RGO.

\subsubsection{Graphene-Titanium Dioxide Nanocomposite (GTNC) Synthesis}

In this study, two GTNC samples were prepared using two different methods, and the effect on the resulting nanocomposite of using a Y-shaped micro-reactor in the green one-pot synthesis method [26] was studied. First, graphene sheets and spherical nano-titanium dioxide particles were ground using an agate mortar and pestle to avoid specimen contamination. Then, the same amount of each powder (i.e., the mass ratio of graphene (RGO) to titanium dioxide was 1:1) was separately dissolved in $50 \mathrm{ml}$ of absolute ethanol using an ultrasonic probe with $500 \mathrm{~W}$ of power, a frequency of $20 \mathrm{kHz}$ and an amplitude of $40 \%$ to achieve a homogeneous dispersion of each powder in solution. The two resulting solutions were then mixed and placed in an ultrasonic bath operating at $160 \mathrm{~W}$ of power and a frequency $40 \mathrm{of} \mathrm{kHz}$ for approximately $90 \mathrm{~min}$. In this step, titanium dioxide molecules adhered to the surfaces of the graphene sheets due to the presence of active sites formed by hydroxyl functional groups on the graphene surface. The resulting solution, which was dark black in colour, as shown in Fig. (1), and was then dried under ambient conditions overnight. The resulting powder was then subjected to microwave irradiation to achieve better exfoliation. The nanocomposite produced using this method was dark black in colour and is referred to as GTNC sample (1).

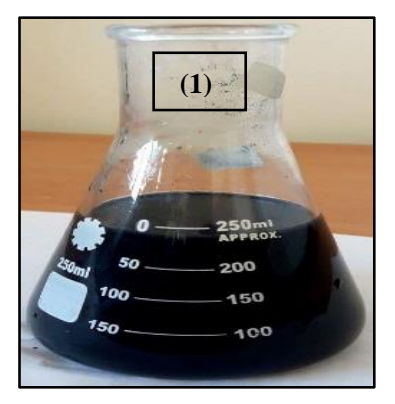

Fig. (1) Black GTNC sample (1) in ethanol solution, produced without the use of a micro-reactor.

GTNC sample (2) was prepared by grinding graphene and titanium dioxide powders and then placing the same amount of each in $50 \mathrm{ml}$ of absolute ethanol and sonicating with an ultrasonic probe for $15 \mathrm{~min}$. The two solutions were pumped at the same time at the optimal flow rate to give the solutions sufficient time to achieve close contact and to avoid any obstruction in the system due to particle accumulation. The solutions were pumped with a syringe pump into a Y-shaped micro-reactor with an estimated channel diameter of $706 \mu \mathrm{m}$ which is the common diameter size used in micro-mixers, as shown in Fig. (2). The small channel diameter of the micro-reactor increased the surface contact between the two powders, maximizing the 
loading and achieving a homogeneous distribution of titanium dioxide nanoparticles on the surfaces of the graphene sheets.

The resulting grey solution obtained from the micro-reactor, which is shown in Fig. (3), was then placed in an ultrasonic bath for $90 \mathrm{~min}$. Then, the sample was dried overnight at ambient temperature. The resulting powder was then subjected to microwave irradiation for better exfoliation. The nanocomposite produced with this method was dark grey in colour and is referred to as GTNC sample (2). A schematic diagram of the new green micro-synthesis method that was used to produce GTNC sample (2) is presented in Fig. (4). To check the repeatability of our novel method, GTNC sample (3) was also prepared using the same process. TEM and EDX analyses of the sample were carried out to confirm the homogeneity of the distribution and the better loading of titanium dioxide nanoparticles on the graphene surfaces.

\begin{tabular}{|c|c|c|c|}
\hline Micro-Reactor Type & $\begin{array}{c}\text { Microscopic } \\
\text { Representation }\end{array}$ & Actual Image & $\begin{array}{c}\text { Average Channel } \\
\text { Diameter }(\boldsymbol{\mu m})\end{array}$ \\
\hline Y-shaped micro-reactor & - & & $706 \mu \mathrm{m}$ \\
\hline
\end{tabular}

Fig. (2) Microscopic representation and actual image of the Y-shaped micro-reactor used in this research.

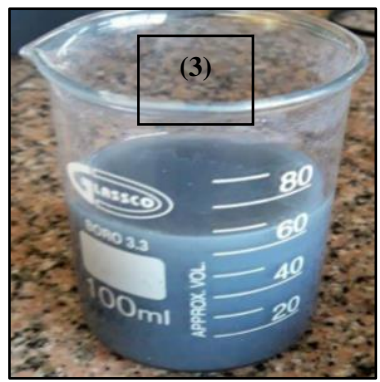

Fig. (3) Grey GTNC sample (2) in ethanol solution, produced using our novel approach.

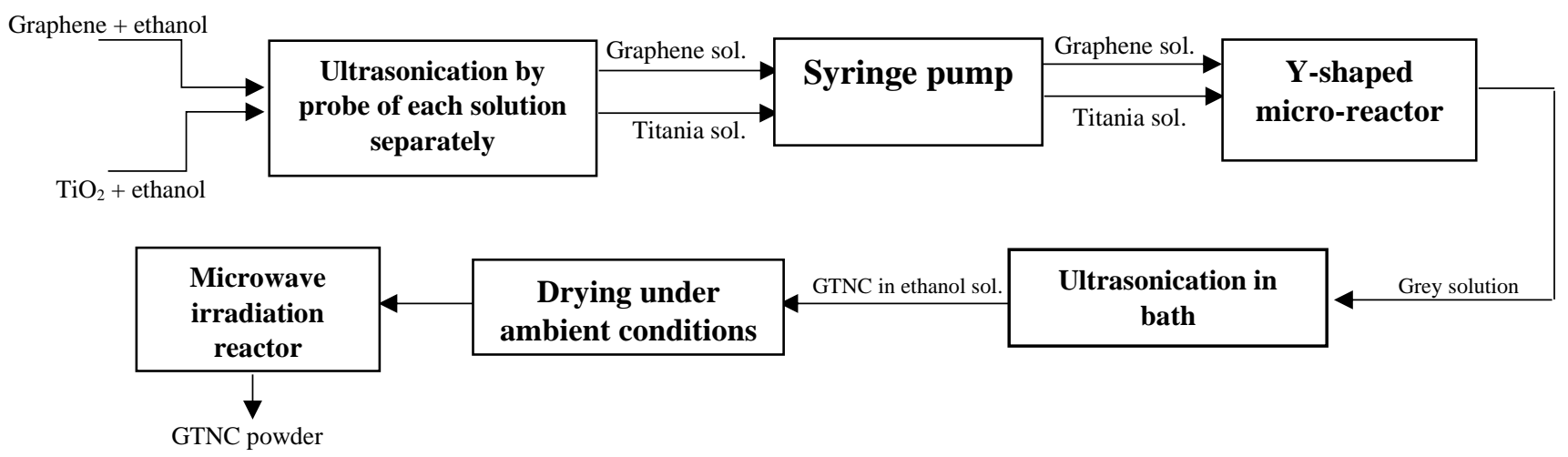

Fig. (4) Schematic diagram of the proposed novel technique for the green micro-synthesis of GTNCs. 


\subsection{Nanoparticles characterization}

\subsubsection{Transmission Electron Microscopy (TEM)}

A field-emission transmission electron microscope (JEOL JEM-2100F) was used in this research. Ethanol was used to dilute the samples by ultrasonication, and the samples were then placed on a grid to dry overnight and were then investigated via TEM after drying. TEM is used to image specimen at a very high resolution using a small wave length electron beam to be able to identify the shape, distribution, size, surface morphology and degree of crystallinity of the nanoparticles.

\subsubsection{Energy-Dispersive X-ray Spectroscopy (EDX)}

EDX was performed using an energy-dispersive X-ray spectroscope (JEOL JEM-2100F) to provide an elemental analysis of the chemical compositions of the samples based on the interactions between excitation X-rays and each sample as each element has specific atomic structure that gives a unique set of peaks on the electromagnetic emission spectrum, it represents a quantitative analysis of high accuracy. The Cliff Lorimer ratio method for thin specimens was used as the quantitation method.

\subsubsection{X-ray Diffraction Spectroscopy (XRD)}

The XRD technique was used to identify the material and determine the crystallinity, unit cell dimensions and chemical properties of the materials. This technique depends on the angles of diffraction of X-ray emissions. The sample must be ground very hard into fine powder before examination. An X-ray diffractor (XRD-6100 Shimadzu, Japan) equipped with a $\mathrm{Cu}-\mathrm{K} \alpha$ radiation source with a wavelength of $\lambda=1.5418 \AA$ was used to perform a 2 theta scan ranging from $5^{\circ}$ to $80^{\circ}$ at a scanning pitch of $0.02^{\circ}$.

\subsubsection{Fourier Transform Infrared Spectroscopy (FTIR)}

FTIR was used to determine and specify the functional groups present in each specimen. Technique depends on infrared spectrum of absorption or emission of the sample, the FTIR spectrometer simultaneously collects high-spectral-resolution data over a wide spectral range and analyse it. A Vertex 70 IR spectrometer (Bruker Scientific Instruments, Germany) was used.

\subsection{Photo-Electrochemical Measurements of Graphene-Titanium Dioxide Nano-Composite}

Photo-electrochemical characterization was conducted in a single-compartment cell with a Pyrex window using a threeelectrode configuration in the presence of $0.5 \mathrm{M} \mathrm{Na}_{2} \mathrm{SO}_{4}$ electrolyte, we used the same set up that was used before in our previous research [29]. GTNC sample (1) and GTNC sample (2) were deposited on fluorine-doped tin oxide (FTO) 
substrates via electrophoretic deposition (EPD) to obtain sample electrodes. In each measurement, one of these sample electrodes was used as the working electrode, a Pt rod was used as the counter electrode, and an $\mathrm{Ag} / \mathrm{AgCl}$ electrode was used as the reference electrode. LSV and chronoamperometry (CAM) were performed using a potentiostat (Princeton Applied Research PARSTAT 4000A). The light irradiation source was a $365 \mathrm{~nm}$ Suruga Seiki UV-LED with an intensity of up to $50 \mathrm{~mW} / \mathrm{cm}^{2}$. The powders of GTNC samples (1) and (2) were deposited on FTO conducting glass via EPD for thin film fabrication. The EPD process was performed using $1 \mathrm{mg}$ of GTNC powder suspended in $50 \mathrm{ml}$ of acetone in the presence of $10 \mathrm{mg}$ of iodine by means of ultrasonication for $15 \mathrm{~min}$. Two parallel FTO electrodes $\left(2.5 \times 2 \mathrm{~cm}^{2}\right)$ were immersed in the solution, and a bias of $50 \mathrm{~V}$ was applied between the electrodes for $20 \mathrm{~min}$ by a DC power supply (Takasago ZX-400LA). The coated area was controlled to be $4 \mathrm{~cm}^{2}$, and the fabricated electrodes were dried in air without any further treatment.

\section{Results and Discussion}

\subsection{Transmission Electron Microscopy (TEM)}

TEM images were used to identify the shape, distribution, size and degree of crystallinity of the nanoparticles. TEM images of the titanium dioxide indicate the formation of perfectly sphere-shaped particles with a high degree of crystallinity, as shown by the image in Fig. (5a), which was acquired at $1 \mathrm{~nm}$ magnification scale. Additionally, the crystallites shown are perfectly stacked, forming a crystalline arrangement. The particle size, as indicated in Fig. (5b) at a magnification scale of $10 \mathrm{~nm}$, ranges from $9 \mathrm{~nm}$ to $25 \mathrm{~nm}$, meaning that the minimal particle size was achieved by this method; this was accomplished by raising the hydrolysis and calcination temperatures. Graphene (RGO) is shown in Figs. (5c) and (5d) at a magnification scale of $100 \mathrm{~nm}$. It is observed that exfoliated graphene sheets formed with a few wrinkles at the edges and that the RGO surfaces have more defects than typical graphene does, causing the RGO sheets to have more reactive sites due to the presence of functional groups on their surfaces. As seen from Figs. (5e) and (5f), the GTNC prepared via the green one-pot synthesis method is not homogeneous, and the titania nanoparticles are not uniformly distributed. Moreover, the loading of titania particles on the graphene sheets is very low, indicating that a significant number of titanium dioxide nanoparticles were wasted, as few dark particles, which correspond to the titanium dioxide particles, are present on the light sheets, which correspond to the graphene sheets. On the other hand, Figs. (5g) and (5h) show that in GTNC sample (2), which was prepared with the aid of the Y-shaped micro-reactor, the titania nanoparticles are homogeneously and uniformly distributed on the graphene surfaces. Indeed, in some areas, the graphene sheet is not visible due to the dense packing of titania on its surface, indicating high loading of titania on the graphene surfaces. Additionally, the TEM images of GTNC sample (3) in Figs. (5i) and $(\mathbf{5} \mathbf{j})$ show good repeatability of our novel synthesis method because GTNC sample (3) also has a very uniform and homogenous distribution of titania particles, similar to that observed in sample (2). GTNC samples (2) and (3), which were 
prepared with our method, show improved crystallographic properties compared with those of GTNC sample (1), which was prepared with the original green one-pot synthesis method. The main cause of the homogenous distribution and high titania content of GTNC sample (2) as shown in the TEM images is that the adherence of the nano-titania particles on the graphene surfaces depends on two types of bonds: chemical bonds with the trace oxygen functionalized groups resulting from the GO preparation step and van der Waals attraction forces. The use of the small-diameter micro-reactor in our novel method considerably enhances the contact between the titania nanoparticles and the graphene sheets by increasing the contact time and contact surface area, which gives the particles sufficient time to diffuse from the solution to the solid surfaces and to form more van der Waals attraction bonds between the titania and graphene and more strong chemical bonds with the remaining
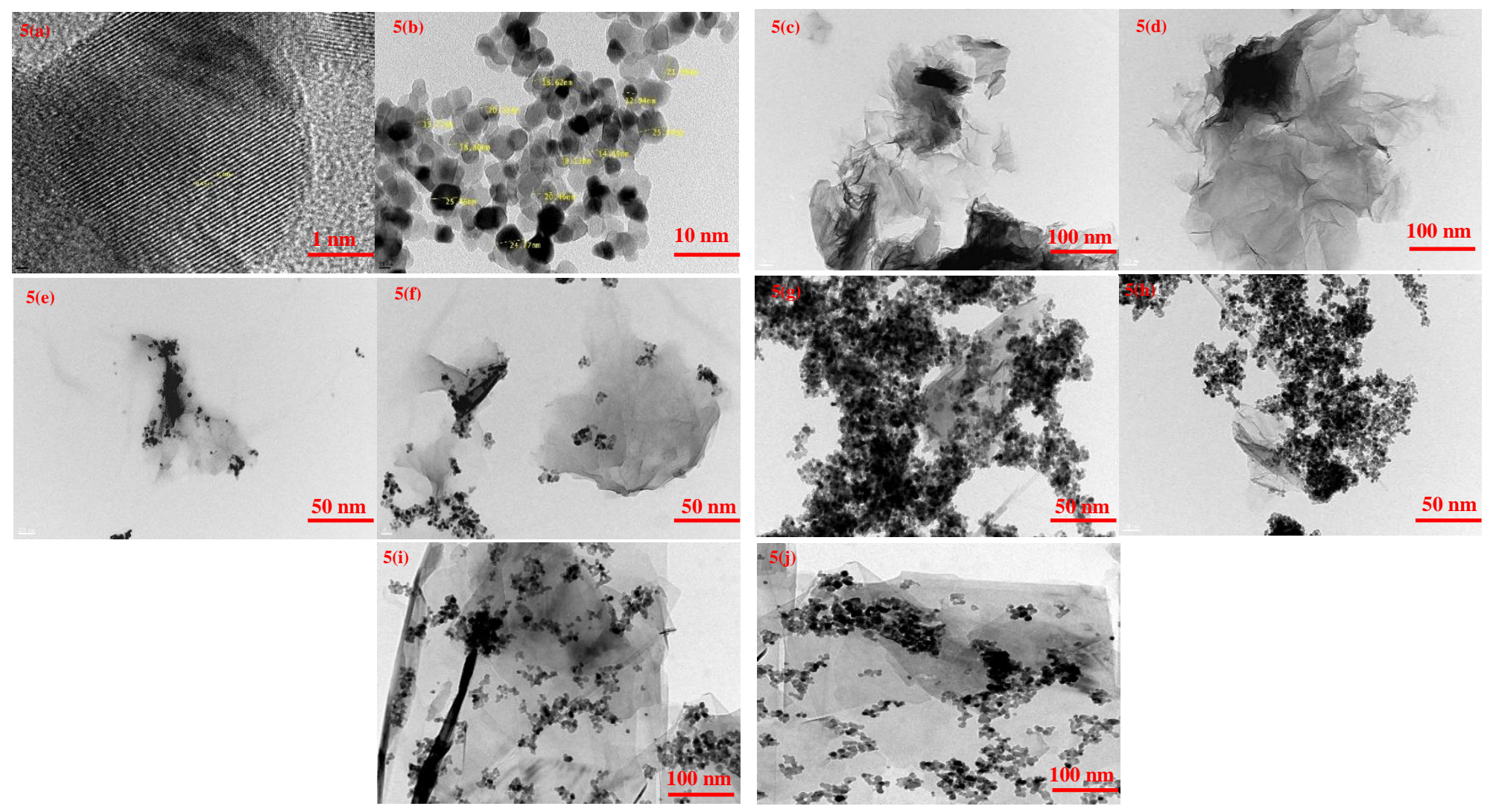

Fig. (5) TEM images of nanoparticles. (a) \& (b) Anatase nano-titanium dioxide at magnification scales of $10 \mathrm{~nm}$ and $1 \mathrm{~nm}$, respectively. (c) \& (d) Graphene (RGO) at a magnification scale of $100 \mathrm{~nm} .(e) \&(f)$ GTNC sample (1) at a magnification scale of $50 \mathrm{~nm} .(\mathrm{g}) \&(\mathrm{~h})$ GTNC sample (2) at a magnification scale of $50 \mathrm{~nm}$. (i) \& (j) GTNC sample (3) at a magnification scale of $100 \mathrm{~nm}$.

\subsection{Energy-Dispersive X-ray Spectroscopy (EDX)}

Figs. (6) and (7) show the EDX spectra and the studied areas for nano-titanium dioxide, graphene (RGO), GTNC sample (1), GTNC sample (2) and GTNC sample (3). In Fig. (8), the EDX results for anatase nano-titanium dioxide indicate that the composition of the formed material is $35.4 \%$ titanium and $64.6 \%$ oxygen by weight. For graphene (RGO), the EDX results 
indicate that $81.3 \%$ of the RGO prepared via chemical exfoliation in this study consists of carbon, with a relatively low oxygen-to-carbon atomic ratio of 0.172 . The oxygen-to-carbon atomic ratio of synthesized graphene typically ranges from 0.041 to 0.18 , as mentioned in previous studies by Mumuera et al. [30], and the remaining functionalized oxygen groups will contribute to the formation of bonds with titania in the nanocomposite. According to the EDX results, GTNC sample (2) has a higher elemental content of titanium than GTNC sample (1) does; the elemental mass fraction of titanium in sample (1) is $0.4 \%$, whereas that in sample (2) is $13.1 \%$. These findings indicate that the titanium dioxide content of GTNC sample (2) is higher than that of GTNC sample (1) by $96.6 \%$, which can be explained by the better nucleation and growth of the titania particles on the graphene that was achieved with the use of the Y-shaped micro-reactor. Finally, the repeatability of our novel method is again confirmed by the EDX results for GTNC sample (3), which is found to contain 79.8\% C, 16.7\% Ti and $12.5 \%$ $\mathrm{O}$; these values are very close to those obtained for GTNC sample (2), confirming the repeatability of our process.
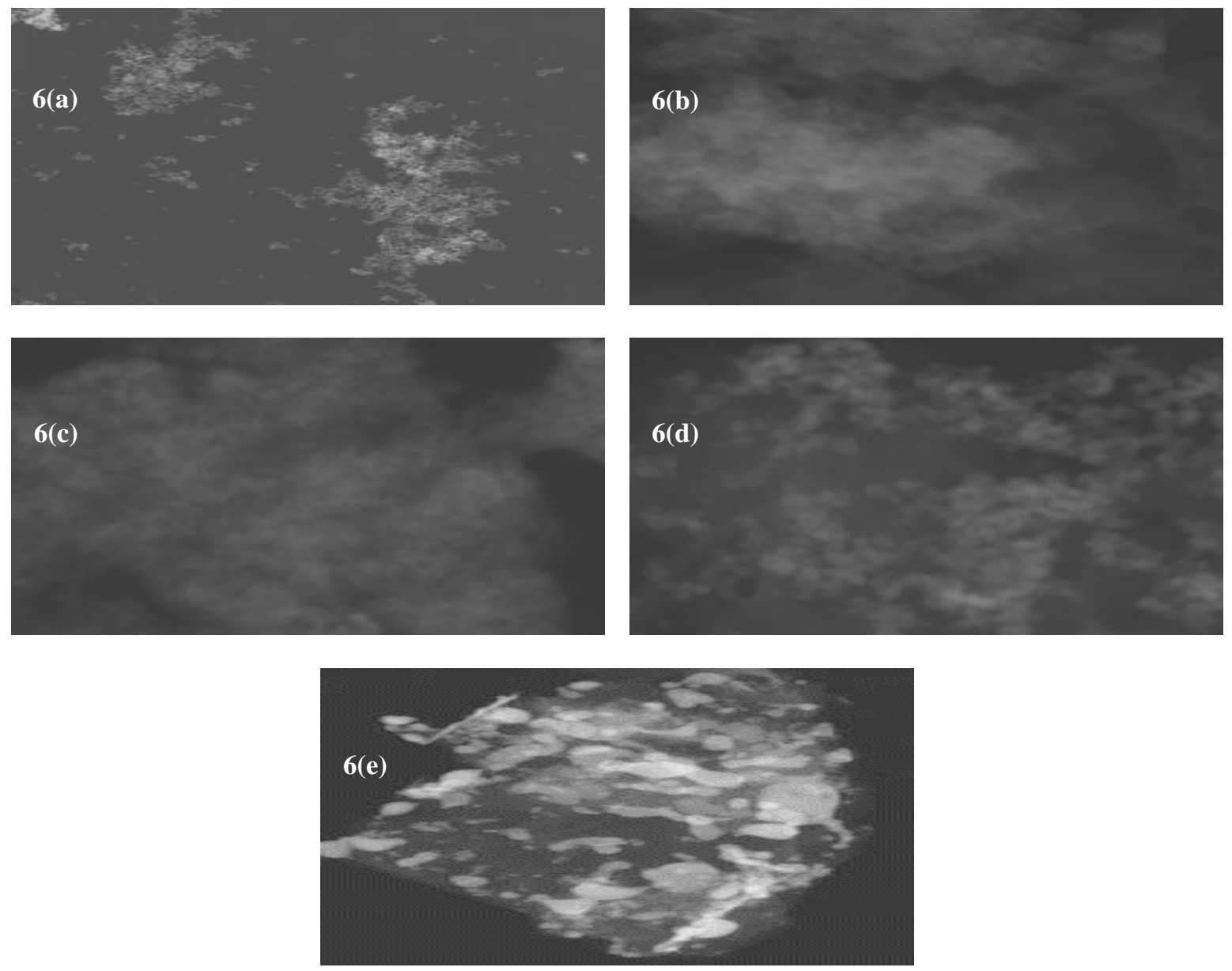

Fig. (6) Areas studied (Electron Image) with EDX for the nanomaterials at $300 \mathrm{~nm}$ scale. (a) Anatase nano-titanium dioxide. (b) Graphene (RGO). (c) GTNC sample (1). (d) GTNC sample (2). (e) GTNC sample (3). 

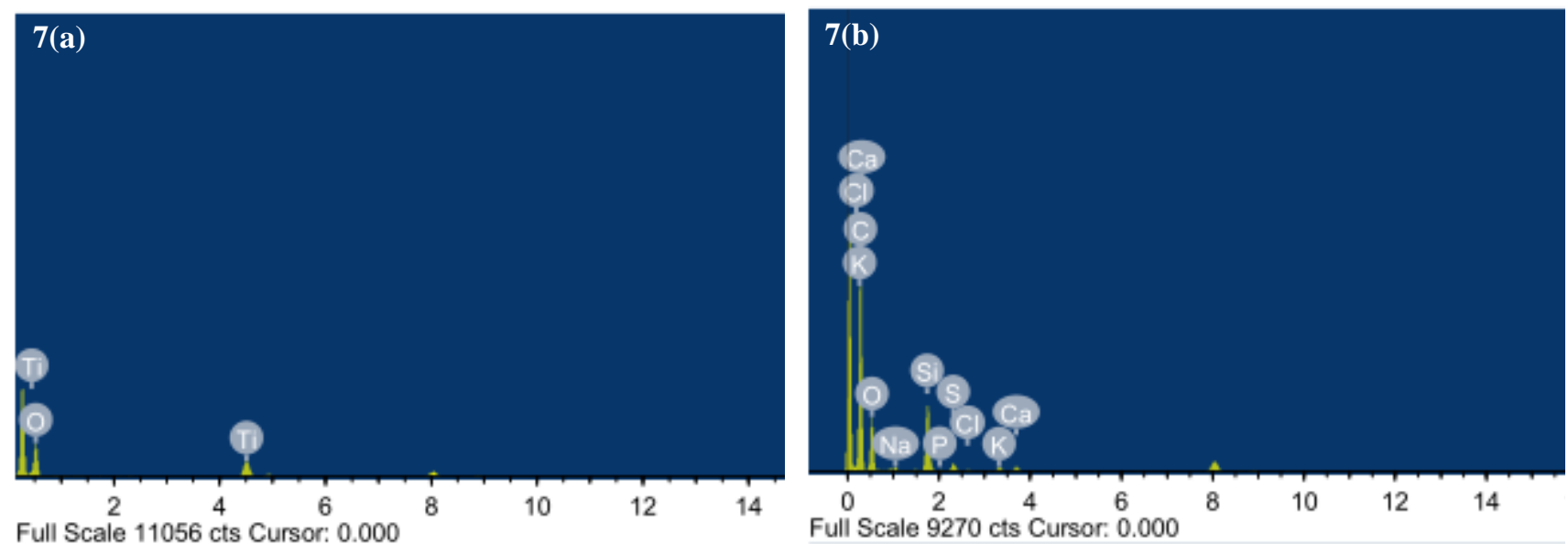

Full Scale 11056 cts Cursor: 0.000
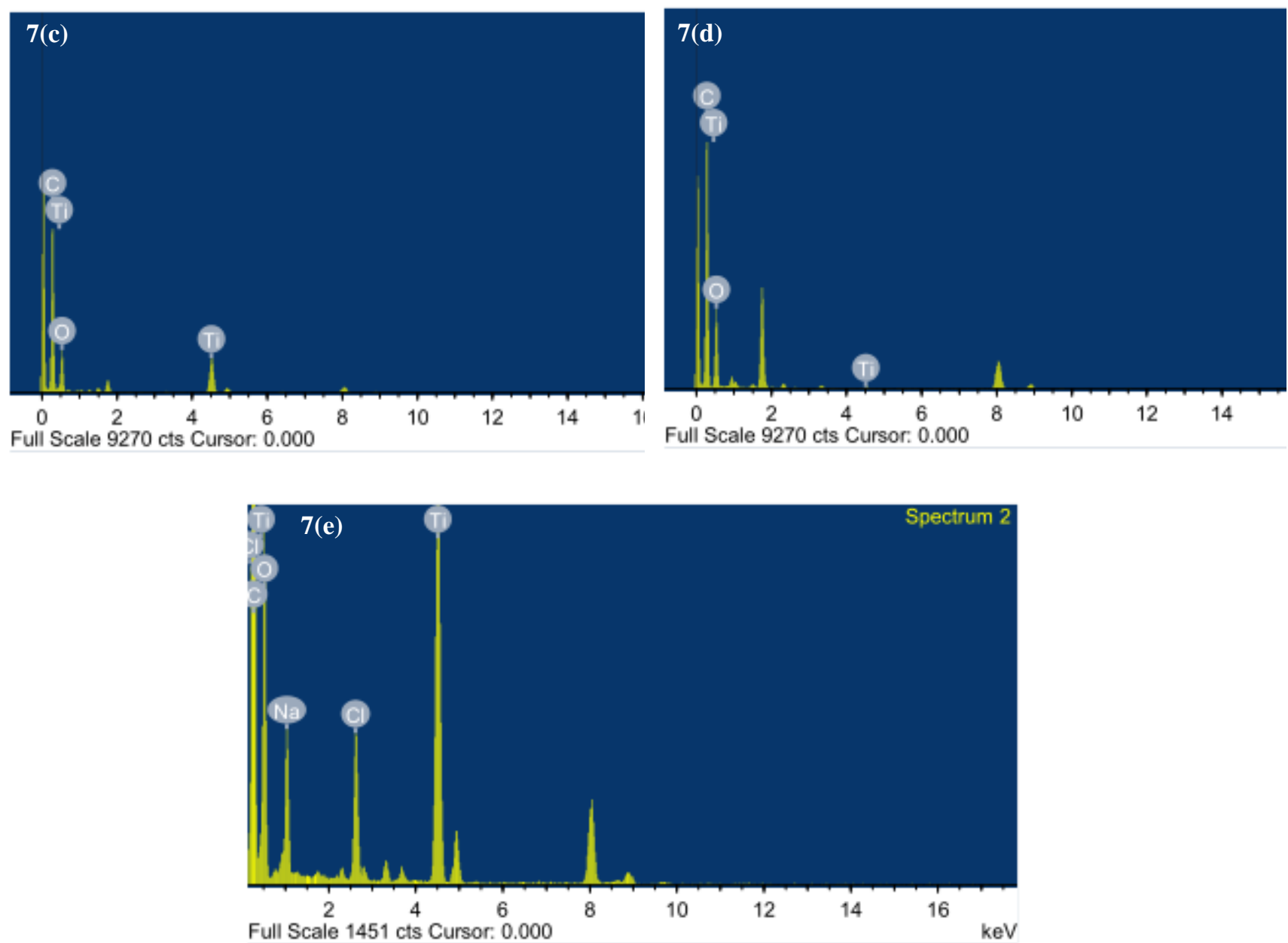

Fig. (7) EDX spectra of the nanomaterials. (a) Anatase nano-titanium dioxide. (b) Graphene (RGO). (c) GTNC sample (1). (d) GTNC sample (2). (e) GTNC sample (3). 


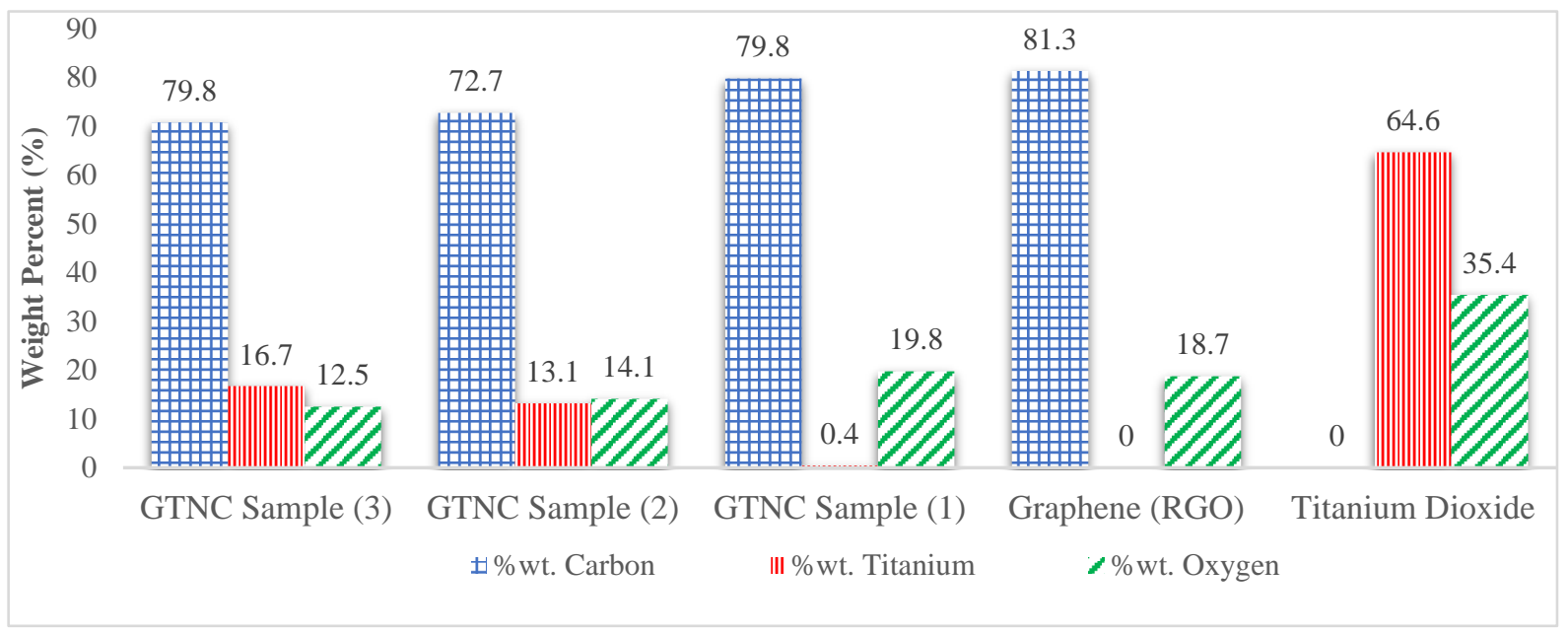

Fig. (8) Elemental weight percentages for anatase nano-titanium dioxide, graphene (RGO), GTNC sample (1), GTNC sample (2) and GTNC sample (3).

\subsection{X-ray Diffraction Spectroscopy (XRD)}

Fig. (9a) indicates that the XRD spectrum of the titania sample matches those of JCPDS card no. 21-1272 and reference [31], confirming the formation of spherical anatase titanium dioxide. Additionally, the highest peak is found at a 2theta value of $25.29^{\circ}$, indicating the formation of titanium dioxide. The peaks at 2 theta values of $25.29^{\circ}, 37.79^{\circ}$ and $48.02^{\circ}$ correspond to the (101), (004) and (200) planes, respectively, and prove the formation of the anatase phase of titanium dioxide with no evidence of the presence of the rutile or brookite phases. The high intensity of the peaks in Fig. (9a) indicates the formation of highly crystalline spherical nano-titania particles. In Fig. (9b), the highest peaks at 2 theta values of $24.38^{\circ}$ and $23.66^{\circ}$ suggest the formation of RGO with relative d-spacings of $0.364 \mathrm{~nm}$ and $0.375 \mathrm{~nm}$, respectively. The d-spacing values corresponding to the highest peaks indicate the formation of a two-dimensional structural layer of graphene (RGO), and the peak at a 2 theta value of $43.3^{\circ}$, corresponding to the (100) plane, reveals the configuration of the hexagonal crystal structure of carbon in the lattice. As seen in Figs. (9c) and (9d), the spectra of both GTNC samples (2) and (1) contain a peak at $25^{\circ}$, which indicates the formation of RGO, and two sharp peaks at $37^{\circ}$ and $48^{\circ}$, which suggest the presence of spherical anatase titanium dioxide. The XRD results show that GTNC samples (1) and (2) have similar diffraction patterns, although with a small difference in the width of the peak at $25^{\circ}$. Table 1 shows the numerical XRD results for the samples along with the corresponding d-spacing, lattice parameters and full width at half maximum (FWHM) for each peak. 


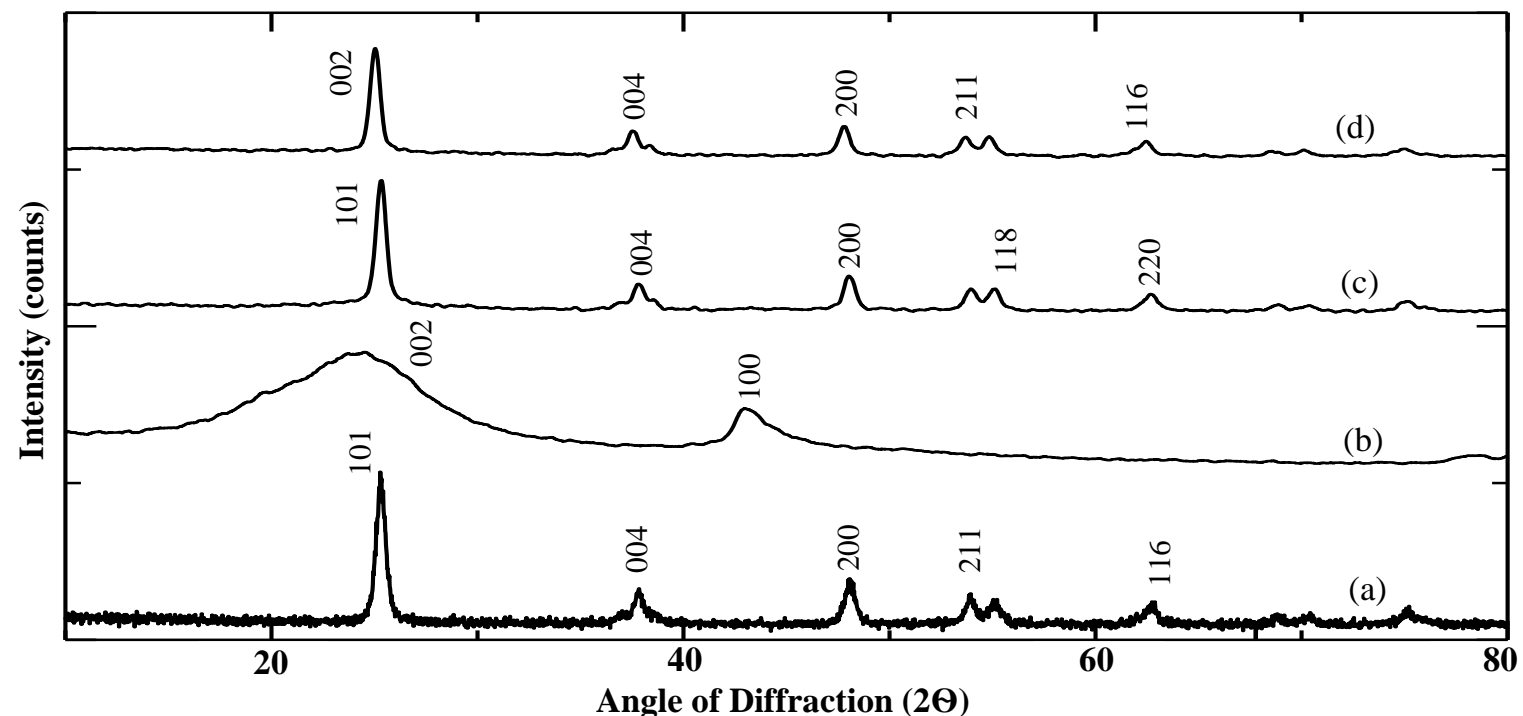

Figure (9) XRD representations. (a) Miller Indices of TiO ${ }_{2}$. (b) Miller Indices of Graphene (RGO). (c) Miller Indices of GTNC sample (2). (d) Miller Indices of GTNC sample (1).

Table 1 Diffraction peak analysis for anatase nano-titanium dioxide, graphene sheets, GTNC sample (1) and GTNC sample (2)

GTNC Sample (1)

GTNC Sample (2)

\begin{tabular}{|c|c|c|c|c|c|c|c|}
\hline 2Theta $\left({ }^{\circ}\right)$ & d-Spacing (A) & FWHM $\left(^{\circ}\right)$ & Miller Indices & 2Theta $\left({ }^{\circ}\right)$ & d-Spacing (A) & FWHM $\left(^{\circ}\right)$ & Miller Indices \\
\hline 24.38 & 3.648 & 0.4 & (002) & 25.01 & 3.557 & 0.56 & (101) \\
\hline 25.29 & 3.517 & 0.57 & (101) & 37.51 & 2.395 & 0.58 & (004) \\
\hline 37.81 & 2.377 & 0.62 & (004) & 47.75 & 1.902 & 0.57 & (200) \\
\hline 48.01 & 1.893 & 0.62 & (200) & 53.64 & 1.707 & 0.57 & (211) \\
\hline 53.93 & 1.698 & 0.64 & (211) & 62.38 & 1.487 & 0.59 & (118) \\
\hline 62.64 & 1.481 & 0.64 & (118) & 70.02 & 1.342 & 0.4 & (116) \\
\hline 70.31 & 1.337 & 0.46 & (116) & 75.22 & 1.262 & 0.42 & (220) \\
\hline 75.92 & 1.252 & 0.28 & (220) & & & & \\
\hline \multicolumn{4}{|c|}{ Anatase Nano-Titanium Dioxide } & \multicolumn{4}{|c|}{ Graphene Sheets (RGO) } \\
\hline 2Theta $\left({ }^{\circ}\right)$ & d-Spacing (A) & FWHM $\left({ }^{\circ}\right)$ & Miller Indices & 2Theta $\left({ }^{\circ}\right)$ & d-Spacing (A) & FWHM $\left({ }^{\circ}\right)$ & Miller Indices \\
\hline 25.29 & 3.518 & 0.5621 & (101) & 24.38 & 3.648 & 0 & $(002)$ \\
\hline 37.79 & 2.378 & 0.5367 & (004) & 41.34 & 2.182 & 0.32 & $(100)$ \\
\hline 48.02 & 1.892 & 0.616 & (200) & & & & \\
\hline 53.89 & 1.699 & 0.56 & (211) & & & & \\
\hline 62.65 & 1.481 & 0.66 & (118) & & & & \\
\hline 70 & 1.342 & 0.38 & (116) & & & & \\
\hline 75.09 & 1.264 & 0.52 & (220) & & & & \\
\hline
\end{tabular}

\subsection{Fourier Transform Infrared Spectroscopy (FTIR)}

As indicated in Fig. (10a), the functional groups observed in the titanium dioxide sample at a wavelength of $732 \mathrm{~cm}^{-1}$ indicate the presence of O-Ti-O anatase bonding. In addition, the peak at $1630.22 \mathrm{~cm}^{-1}$ is associated with water adsorbed on the particle surfaces, whereas the peak at $3444.24 \mathrm{~cm}^{-1}$ shows the presence of hydroxyl groups which matches with peaks indicated in previous research Samira et al. [32], and the peak at $2922 \mathrm{~cm}^{-1}$ indicates the presence of trace amounts of organic 
C-H bonds. Figs. (10b) and (10c) present the FTIR spectra of GTNC samples (2) and (1). Both samples have peaks at almost the same wavelengths corresponding to vibrating bonds, with the peak at $3441.45 \mathrm{~cm}^{-1}$ indicating water adsorbed on the surface, the peak at $2923 \mathrm{~cm}^{-1}$ corresponding to carbon-hydrogen single bonds, the peak at $1633 \mathrm{~cm}^{-1}$ corresponding to hydroxyl group bonds, and the peaks at $1284 \mathrm{~cm}^{-1}, 1121 \mathrm{~cm}^{-1}$ and $1137 \mathrm{~cm}^{-1}$ corresponding to carbon-oxygen single bonds. The FTIR results show that GTNC samples (1) and (2) have the same functional groups adsorbed on their surfaces, namely, C-O, C-H, O-H and $\mathrm{H}_{2} \mathrm{O}$. Fig. (10d) shows the FTIR spectrum of the graphene (RGO), in which the peak at $3453 \mathrm{~cm}^{-1}$ is related to the vibrations of hydroxyl groups, the peak at $1636.7 \mathrm{~cm}^{-1}$ suggests the vibrations of carbon-carbon double bonds, and those at $1384.3 \mathrm{~cm}^{-1}$ and $1116 \mathrm{~cm}^{-1}$ indicate the vibrations of carbon-oxygen single bonds.

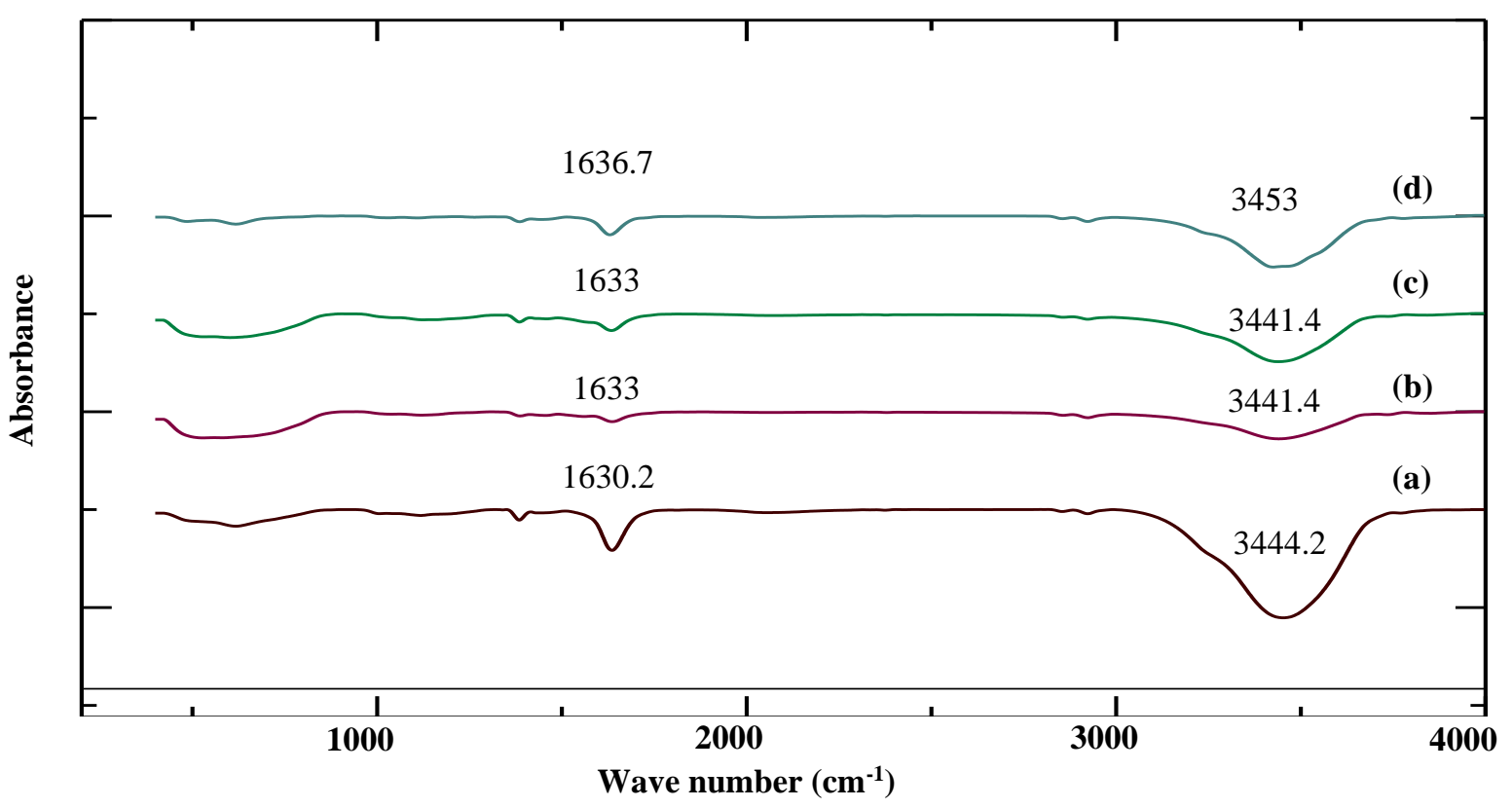

Fig. (10) FTIR representations. (a) $\mathrm{TiO}_{2}$. (b) GTNC sample (2). (c) GTNC sample (1). (d) Graphene (RGO).

\subsection{Photo-Electrochemical Measurements}

CAM was conducted to determine the photocurrent stability of GTNC samples (1) and (2), and the results are displayed in Fig. (11). The tests were performed by applying a bias of $1.17 \mathrm{~V}$ for $800 \mathrm{~s}$ to each sample. Fig. (11) shows the CAM curve for $800 \mathrm{~s}$ at the desired electrochemical potential. Initially, the sample was left for $60 \mathrm{~s}$ in the dark until a steady current was attained. Then, UV-LED light $(365 \mathrm{~nm})$ with a maximum intensity of $50 \mathrm{~mW} \mathrm{~cm}^{-2}$ was applied for $600 \mathrm{~s}$ and then turned off. The findings show that the two electrodes have different curves, which indicate that the electrode prepared with GTNC sample (2) is more stable than that prepared with GTNC sample (1). Moreover, GTNC sample (2) has a higher peak current of 60 $\mu$ A compared with that of GTNC sample (1), which indicates that the separation and transfer of electron-hole pairs are better in the case of GTNC sample (2) (the sample prepared with our novel method) because this sample has a higher loading content of titania particles, as shown by the TEM and EDX results; the combination of titania with a carbonaceous material 
forms a heterojunction nanostructure that restricts the electron-hole pair recombination rate in titania, leading to higher quantum activity and better photocatalytic activity, consistent with previous research Melian et al. [33].

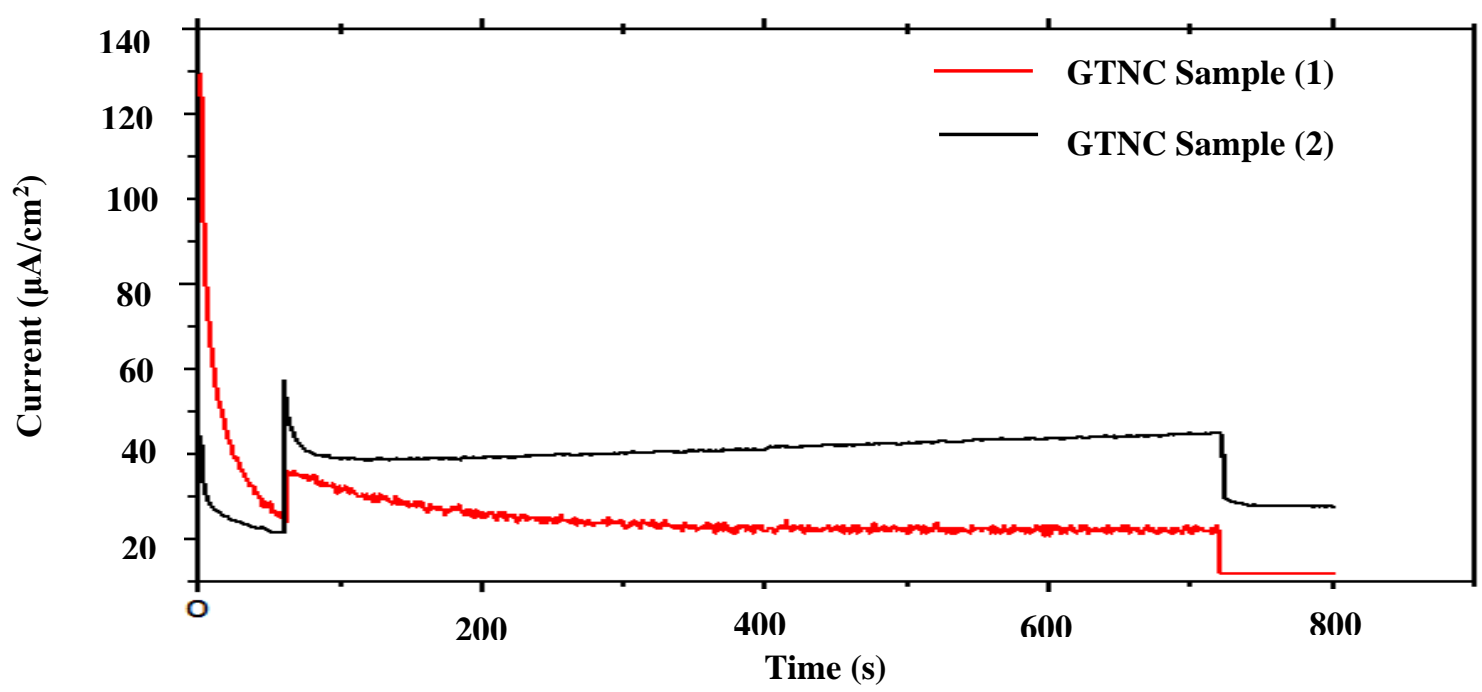

Fig. (11) CAM measurements of GTNC sample (1) and GTNC sample (2) over $800 \mathrm{~s}(60 \mathrm{~s}$ in the dark, then $600 \mathrm{~s}$ under $U V$-LED light $(365 \mathrm{~nm})$ with a maximum intensity of $50 \mathrm{~mW} \mathrm{~cm}^{-2}$, then dark again) at $1.17 \mathrm{Vvs.} \mathrm{Ag/AgCl}$.

LSV was used to investigate the capabilities of the electrodes prepared with GTNC samples (1) and (2). The LSV sweep was conducted from $-1 \mathrm{~V}$ to $+1 \mathrm{~V} v s$. $\mathrm{Ag} / \mathrm{AgCl}$ at a scan rate of $0.05 \mathrm{~V} / \mathrm{s}$ under chopped light irradiation (UV-LED, $365 \mathrm{~nm}$ ). The photocatalytic activity of a semiconductor material depends on the amount of current generated due to the transfer of electrons from the photoanode electrode to the counter electrode under light irradiation. The photocurrent measurements of the GTNC sample (1) and (2) electrodes are shown in Fig. (12). The generation of photocurrent in the samples through multiple on-off cycles is observed, which indicates that the electrodes are stable and that the photocurrent is quite reversible. 


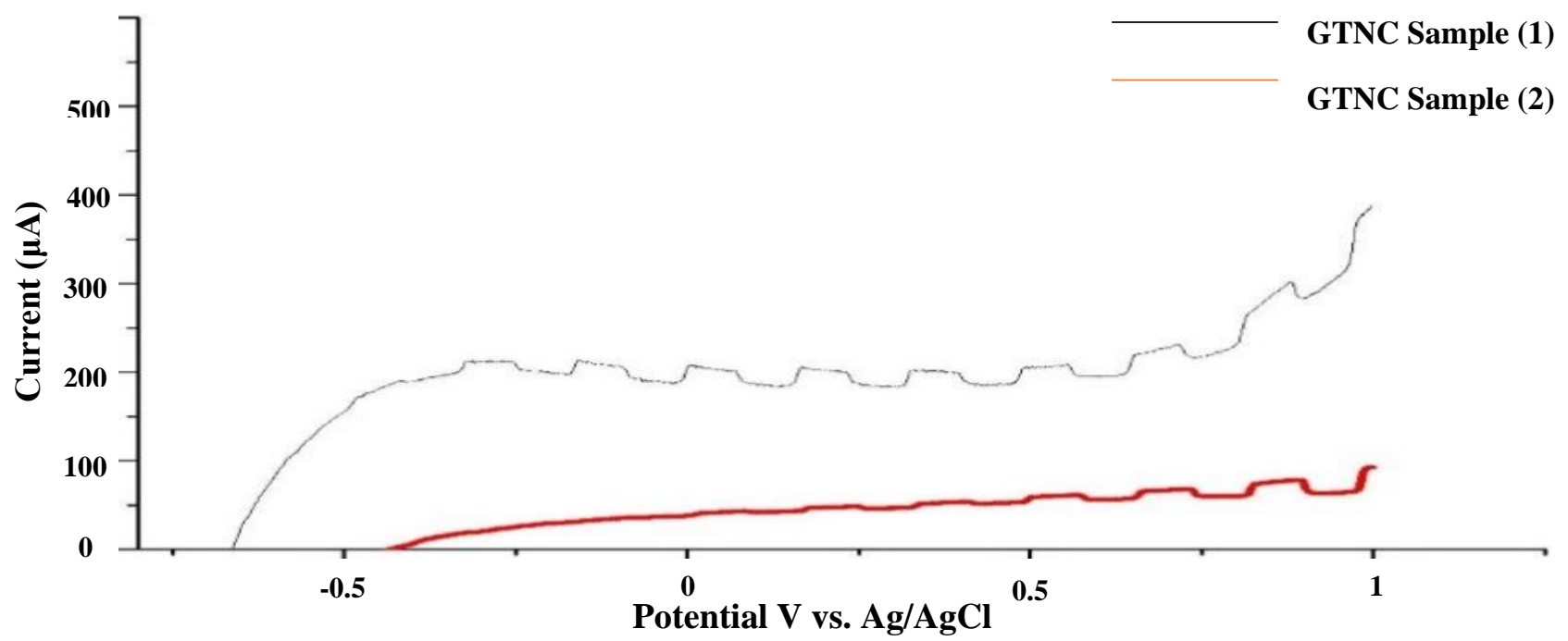

Fig. (12) Linear scan voltammograms of GTNC samples (1) and (2) under dark-light cycles. Measurements were performed

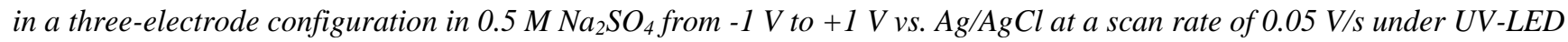
$(365 \mathrm{~nm})$ irradiation.

\section{Conclusion}

- A new methodology has been established for producing graphene-titanium dioxide nanocomposites (GTNCs) with an excellent distribution and a high loading content of titanium dioxide on the graphene sheets.

- High calcination temperature $650^{\circ} \mathrm{C}$ resulted in promoting the formation of spherical nano-titanium dioxide in the anatase phase with very high crystallinity with small particle diameters ranging from $9 \mathrm{~nm}$ to $25 \mathrm{~nm}$.

- The as prepared graphene (RGO) exhibited minimal flake folding and a high carbon content of $81.28 \%$ with a low oxygen-to-carbon atomic ratio of 0.172 .

- GTNC sample (2) produced with this novel approach, shows a $96.6 \%$ increase in titania content over that of GTNC sample (1) and a more homogeneous distribution of spherical anatase titanium dioxide nanoparticles on the graphene (RGO) sheets, which provide it with superior crystallographic and physiochemical properties due to the better nucleation and growth of the titania particles.

- The FTIR results show that GTNC samples (1) and (2) have functional groups of C-O, C-H, O-H and $\mathrm{H}_{2} \mathrm{O}$ adsorbed on their surfaces.

- The XRD results show that GTNC samples (1) and (2) have similar diffraction patterns, with a small difference in the width of the peak at a 2 theta value of $25^{\circ}$.

- The repeatability of our novel process is confirmed by the TEM and EDX analysis of GTNC sample (3). 
- Chronoamperometry measurements indicate the electrode prepared with GTNC sample (2) is more stable than that prepared with GTNC sample (1). Moreover, GTNC sample (2) has a higher peak current of $60 \mu \mathrm{A}$ compared with that of GTNC sample (1), which indicates that the separation and transfer of electron-hole pairs are better in the case of GTNC sample (2). According to the LSV results, the generation of photocurrent in the samples can be observed through multiple on-off cycles, which indicates that the electrodes are stable and that the photocurrent is quite reversible.

\section{List of Abbreviations}

GTNC Graphene Titanium Dioxide Nano Composite

TEM Transmission Electron Microscopy

EDX Energy Dispersive X-ray Spectroscopy

FTIR Fourier Transform Infrared Spectroscopy

XRD X-ray Diffraction Spectroscopy

UV Ultra-Violet

PEC Photo Electro-Chemical

EPD Electrophoretic deposition

FTO Fluorine doped tin oxide

LSV Linear Sweep Voltammetry

CAM Chronoamperometry

\section{Conflict of Interest}

The authors certify that they have NO affiliations with or involvement in any organization or entity with any financial interest (such as honoraria; educational grants; participation in speakers' bureaus; membership, employment, consultancies, stock ownership, or other equity interest; and expert testimony or patent-licensing arrangements), or non-financial interest (such as personal or professional relationships, affiliations, knowledge or beliefs) in the subject matter or materials discussed in this manuscript.

\section{Acknowledgement}

We are grateful to our colleagues from Egypt-Japan University for Science and Technology, Eng. Islam Salama and Eng. Samar Nabil, who provided insight and expertise that greatly assisted in this research.

\section{References}

[1] Novoselov, K.S.; Geim, A.K.; Morozov, S.V.; Jiang, D.; Zhang, Y.; Dubonos, S.V. Grigorieva, I.V.; Firsov, A.A. Electric field effect in atomically thin carbon films. Science, 2004, 306(5696), 666-669. 
[2] Zhu, Y.; Murali, S.; Cai, W.; Li, X.; Suk, J.W.; Potts, J.R.; Ruoff, R.S. Graphene and graphene oxide: Synthesis, properties, and applications. Adv. Mater., 2010, 22(35), 3906-3924.

[3] Huc, V.; Bendiab, N.; Rosman, N.; Ebbesen, T.; Delacour, C.; Bouchiat, V. Large and flat graphene flakes produced by epoxy bonding and reverse exfoliation of highly oriented pyrolytic graphite. Nanotechnology, 2008, $19(45), 455601$.

[4] Francesco, B.; Antonio, L.; Tawfique, H.; Zhipeie, S.; Luigi, C.; Andrea, C.F. Production and processing of graphene and 2d crystals. Mater. Today, 2012, 15(12), 564-589.

[5] Parvez, K.; Wu, Z.S.; Li, R.; Liu, X.; Graf, R.; Feng, X.; Mullen, K. Exfoliation of graphite into graphene in aqueous solutions of inorganic salts. J. Am. Chem. Soc., 2014, 136(16), 6083-6091.

[6] Hernandez, Y.; Nicolosi, V.; Lotya, M.; Blighe, F.M.; Sun, Z.; De, S.; McGovern, I.T.; Holland, B.; Byrne, M.; Gun'Ko, Y.K.; Boland, J.J.; Niraj, P.; Duesberg, G.; Krishnamurthy, S.; Goodhue, R.; Hutchison, J.; Scardaci, V.; Ferrari, A.C.; Coleman, J.N. High-yield production of graphene by liquid-phase exfoliation of graphite. Nat. Nanotechnol., 2008, 3(9), 563568.

[7] Warner, J.H.; Schaffel, F.; Rummeli, M.; Bachmatiuk, A. Graphene: Fundamentals and emergent applications; Newness. Technology \& Engineering, 2012.

[8] Zhou, M.; Wang, Y.; Zhai, Y.; Zhai, J.; Ren, W.; Wang, F.; Dong, S. Controlled synthesis of large-area and patterned electrochemically reduced graphene oxide films. Chem. Eur. J., 2009, 15, 6116-6120.

[9] Kim, K.S.; Zhao, Y.; Jang, H.; Lee, S.Y.; Kim, J.M.; Kim, K.S.; Ahn, J.H.; Kim, P.; Choi, J.Y.; Hong, B.H. Large-scale pattern growth of graphene films for stretchable transparent electrodes. Nature, 2009, 457(7230), 706-710.

[10] Strudwick, A.J.; Weber, N.E.; Schwab, M.G.; Kettner, M.; Weitz, R.T.; Wünsch, J.R.; Mullen, K.; Sachdev, H. Chemical vapour deposition of high-quality graphene films from carbon dioxide atmospheres. ACS Nano, 2014, 9(1), 31-42.

[11] Wen, T.; Gao, J.; Shen, J.; Zhou, Z. Preparation and characterisation of $\mathrm{TiO}_{2}$ thin films by the sol-gel process. J. Mater.

Sci., 2001, 36(24), 5923-5926.

[12] Sharmila, D.R.; Venkatesh, R.; Sivaraj, R. Synthesis of titanium dioxide nanoparticles by sol-gel technique. Int. J. Innov. Res. Sci. Eng. Technol., 2014, 3(8), 15206-15211.

[13] Niederberger, M.; Bartl, M.H.; Stucky, G.D. Benzyl alcohol and titanium tetrachloride: A versatile reaction system for the non-aqueous and low-temperature preparation of crystalline and luminescent titania nanoparticles. Chem. Mater., 2002, 14(10), 4364-4370.

[14] Tacchini, I.; Ansón, C.A.; Yu, Y.; Martinez, M.T.; Lira, C.M. Hydrothermal synthesis of 1D TiO 2 nanostructures for dye-sensitised solar cells. Mater. Sci. Eng. B, 2012, 177(1), 19-26. 
[15] Dinh, C.T.; Nguyen, T.D.; Kleitz, F.; Do, T. Shape-controlled synthesis of highly crystalline titania nanocrystals. ACS Nano, 2009, 3(11), 3737-3743.

[16] Goel, P.; Yadav, K.L. A comparative analysis of PBZT synthesised by coprecipitation and sol-gel method. Indian J. Eng. Mater. Sci., 2005, 12, 552-556.

[17] Aydin, F.A.; Soylak, M. A novel multi-element coprecipitation technique for separation and enrichment of metal ions in environmental samples. Talanta, 2007, 73(1), 134-141.

[18] Shimizu, T.; Ito, H.; Kawaguchi, H.; Shijo, Y. Determination of trace molybdenum in water samples by electrothermal atomic absorption spectrometry after pre-concentration with miniaturised coprecipitation. Bull. Chem. Soc. Japan, 1999, 72(1), 43-46.

[19] Akagi, T.; Haraguchi, H. Simultaneous multielement determination of trace metals using ten $\mathrm{mL}$ of seawater by inductively coupled plasma atomic emission spectrometry with gallium coprecipitation. Anal. Chem., 1990, 62(1), 81-85.

[20] Yu, J.C.; Yu, J.; Ho, W.; Zhang, L. Preparation of highly photocatalytic active nano-sized $\mathrm{TiO}_{2}$ particles via ultrasonic irradiation. Chem. Commun., 2001, 7(19), 1942-1943.

[21] Stefan, S.; Markus, W.; Horst, H. Nanocrystalline titania films and particles by chemical vapor synthesis. Chem. Vapor Depos., 2000, 6(5), 239-244.

[22] Xiaobo, C.; Samuel, S.M. Titanium dioxide nanomaterials: Synthesis, properties, modifications, and application's. Chem. Rev., 2007, 107, 2891-2959.

[23] Wojtoniszak, M.; Zielinska, B.; Chen, X.; Kalenczuk, R.J.; Borowiak, P.E. Synthesis and photocatalytic performance of $\mathrm{TiO}_{2}$ nanospheres-graphene nanocomposite under visible and UV light irradiation. J. Mater. Sci., 2012, 47(7), 3185-3190. [24] Zhang, X.; Sun, Y.; Cui, X.; Jiang, Z. A green and facile synthesis of $\mathrm{TiO}_{2} /$ graphene nanocomposites and their photocatalytic activity for hydrogen evolution. Int. J. Hydrogen Energy, 2012, 37(1), 811-815.

[25] Chin, W.L.; Foo, W.L.; Soon, W.C.; Christelle, P.P.; Siti, Z.B.; Joon, C.J.; Sharifah, B. An overview: Recent development of titanium dioxide loaded graphene nanocomposite film for Solar application. Curr. Org. Chem., 2015, 19, $1882-1895$.

[26] Dey, A.; Nangare, V.M.; Khan, M.A.S.; Khanna, P.K.; Skider, A.K.; Chattopadhyay, S. A graphene titanium dioxide nanocomposite (GTNC): One pot green synthesis and its application in a solid rocket propellant. RSC Adv., 2015, 5, 6377763785.

[27] Kiarii, E.; Govender, K.; Ndungu, P.; Govender, P.P. Recent advances in titanium dioxide/graphene photocatalyst materials as potentials of energy generation. Bull. Mater. Sci., 2018, 41, 75. 
[28] Li, W.; Zeng, T. Preparation of $\mathrm{TiO}_{2}$ anatase nanocrystals by $\mathrm{TiCl}_{4}$ hydrolysis with additive $\mathrm{H}_{2} \mathrm{SO}_{4}$. Plos One, 2011, 6(6), 21082.

[29] Hammad, A.S.; Haitham, M.B.; El-Shazly, A.H.; Elkady, M.F. Effect of $\mathrm{WO}_{3}$ morphological structure on its photoelectrochemical properties. Int. J. Electrochem. Sci., 2018, 13, 362-372.

[30] Munuera, J.M.; Paredes, J.I.; Villar-Rodil, S.; Ayán-Varela, M.; Pagán, A.; Aznar-Cervantes, S.D.; Cenis, J.L.; MartínezAlonso, A.; Tascón, J.M.D. High quality, low oxygen content and biocompatible graphene nanosheets obtained by anodic exfoliation of different graphite types. Carbon, 2015, 94, 729-739.

[31] Gopalakrishnan, A.; Binitha, N.N.; Yaakob, Z.; Akbar, P.M.; Padikkaparambil, S. Excellent photocatalytic activity of titania-graphene nanocomposites prepared by a facile route. J. Sol-Gel Sci. Technol., 2016, 80(1), 189-200.

[32] Samira, B.; Kamyar, S.; Sharifah, B.A.H. Synthesis and characterization of anatase titanium dioxide nanoparticles using egg white solution via sol-gel method. J. Chem., 2013, 6, 48205.

[33] Melian, E.P.; Lopez, C.R.; Mendez, A.O.; Diaz, O.G.; Suarez, M.N.; Rodriguez, J.M.D.; Navio, J.A.; Hevia, D.F. Hydrogen production using Pt-loaded $\mathrm{TiO}_{2}$ photocatalysts. Int. J. Hydrogen Energy, 2013, 38, 11737. 